\title{
IncRNA transcription factor 7 is related to deteriorating clinical features and poor prognosis in multiple myeloma, and its knockdown suppresses disease progression by regulating the miR-203-mediated Jagged1-Notch1 signaling pathway
}

\author{
HAIYAN LIU ${ }^{1,2}$, YAODONG SHEN ${ }^{2}$, YA XU $^{2}$, LI WANG $^{2}$, CHENLU ZHANG $^{2}$, \\ YIJING JIANG ${ }^{2}$, LEMIN HONG ${ }^{2}$, HONGMING HUANG ${ }^{2}$ and HONG LIU ${ }^{2}$ \\ ${ }^{1}$ Department of Hematology, The First Affiliated Hospital of Soochow University, Suzhou, Jiangsu 215006; \\ ${ }^{2}$ Department of Hematology, The Affiliated Hospital of Nantong University, Nantong, Jiangsu 226001, P.R. China
}

Received July 31, 2020; Accepted February 11, 2021

DOI: $10.3892 / \mathrm{ol} .2021 .12673$

\begin{abstract}
Multiple myeloma(MM) remains a challenge to treat, and its precise pathogenic mechanisms have not been fully clarified. The present study aimed to evaluate the relation between long non-coding RNA transcription factor 7 (lnc-TCF7) and clinical features, as well as the prognosis of patients with $\mathrm{MM}$, and to determine the effects of lnc-TCF7-knockdown on the regulation (and regulatory mechanisms) of MM progression. lnc-TCF7 expression was detected in the bone marrow plasma cells of 86 patients with MM and 30 healthy controls. In patients with MM, the clinical data were collected, and event-free survival (EFS) and overall survival (OS) analyses were conducted. In vitro, lnc-TCF7 expression was detected in $\mathrm{MM}$ cell lines and normal bone marrow plasma cells. Using Roswell Park Memorial Institute 8226 cells, functional experiments were conducted following lnc-TCF7 short hairpin (sh)RNA transfection, and compensation experiments
\end{abstract}

Correspondence to: Professor Hong Liu or Professor Hongming Huang, Department of Hematology, The Affiliated Hospital of Nantong University, 20 Xisi Road, Nantong, Jiangsu 226001, P.R. China

E-mail: hongliu63@163.com

E-mail: hhmmmc@163.com

Abbreviations: MM, multiple myeloma; lncRNAs, long non-coding RNAs; lnc-TCF7, lncRNA transcription factor 7; CRC, colorectal cancer; NSCLC, non-small cell lung cancer; BMMCs, bone marrow mononuclear cells; $\beta 2-\mathrm{MG}, \beta-2$-microglobulin; ISS, International Staging System; qPCR, quantitative polymerase chain reaction; $\mathrm{CR}$, complete response; VGPR, very good partial response; ORR, overall response rate; EFS, event-free survival; OS, overall survival; RPMI, Roswell Park Memorial Institute; AUC, area under the curve; ROC, receiver operating characteristic; ceRNA, competing endogenous RNA; miRNAs, microRNAs

Key words: multiple myeloma, lnc-TCF7, miR-203, Jagged1, Notch1 were performed after lnc-TCF7 shRNA transfection alone and in combination with a microRNA (miR)-203 inhibitor. lnc-TCF7 expression was increased in patients with MM compared with the healthy controls and was positively related to $\beta$-2-microglobulin expression and International Staging System stage, while negatively associated with complete response, EFS and OS. In vitro, lnc-TCF7 was upregulated in MM cells compared with normal bone marrow plasma cells, and its knockdown suppressed MM cell proliferation while promoting apoptosis. Compensation experiments showed that miR-203 inhibition promoted MM progression by regulating the Jagged1-Notch1 signaling pathway in lnc-TCF7-knockdown cells. In conclusion, increased lnc-TCF7 expression was related to deteriorating clinical features and prognosis, and lnc-TCF7-knockdown inhibited disease progression by regulating the miR-203-mediated Jagged1-Notch1 signaling pathway activation in MM.

\section{Introduction}

Multiple myeloma (MM), also known as plasma cell myeloma, is a fatal hematological malignancy characterized by the malignant proliferation of MM cells and an aberrant increase in monoclonal immunoglobulin (1). In patients with MM, this immunoglobulin excess results in platelet dysfunction, hyperviscosity, bone loss and renal tubular damage, which subsequently leads to bleeding, neurological impairment, ostealgia, renal failure and other life-threatening symptoms (2). According to a recent epidemiological study, there were 159,985 new diagnoses and 106,105 MM-associated deaths worldwide in 2018, accounting for $0.9 \%$ of new cancer cases and $1.1 \%$ of all cancer-associated deaths (3). Currently, the standard treatment strategy for MM is high-dose chemotherapy (such as carfilzomib, bortezomib and melphalan) followed by stem cell transplantation (if accessible), which effectively prolongs patient survival time (4). However, due to its complexity, MM remains a challenge to treat, and its precise pathogenic mechanisms have not been fully clarified $(5,6)$. Therefore, it is important to determine the 
underlying etiology of MM and to identify potential new treatment targets.

lncRNA transcription factor 7 (lnc-TCF7) is a novel lncRNA that was first identified in liver cancer cells, and promotes the self-renewal of human liver cancer stem cells through the activation of Wnt signaling (7). Furthermore, lnc-TCF7 has been discovered to promote the carcinogenesis of various malignancies, including colorectal cancer (CRC) and non-small cell lung cancer (NSCLC) (8-10). Moreover, the TCF7 gene promotes cellular functions, such as self-renewal and differentiation, in hematological malignancies (11). Furthemore, our previous preliminary research indicated that in a small sample population, lnc-TCF7 was upregulated in patients with MM compared with healthy donors, and was also upregulated in MM cell lines compared with plasma cells from healthy human primary bone marrow mononuclear cells (BMMCs) (unpublished data). Thus it was hypothesized that lnc-TCF7 may also facilitate MM development and progression. However, the clinical implications of lnc-TCF7 in MM diagnosis and prognosis, as well as its role in MM progression, remain to be elucidated.

Therefore, the aim of the present study was to evaluate the effects of 1nc-TCF7 on the risk of developing MM, and its influence on clinicopathological features and patient prognosis. The study also sought to further explore the effects of lnc-TCF7-knockdown on the regulation of cell proliferation and apoptosis, as well as its molecular mechanism in MM.

\section{Materials and methods}

Participants. A total of 86 patients with de novo symptomatic MM were consecutively recruited from The Affiliated Hospital of Nantong University (Nantong, China) between July 2014 and December 2017. The inclusion criteria were as follows: i) A diagnosis of de novo symptomatic MM based on the International Myeloma Working Group recommendations for global myeloma care (2013) (12), ii) age $>18$ years old and iii) no history of stem cell transplantation, chemotherapy, radiotherapy or other systematic treatments. Patients were excluded from the study if they presented with other malignancies, had serious infection (such as human immunodeficiency virus), or were pregnant or lactating. In addition, 30 healthy individuals who donated bone marrow at the hospital were enrolled as healthy controls. The median age of the healthy controls was 55 years with a range of 31-74 years. Meanwhile, there were $19(63.3 \%)$ males as well as 11 (36.7\%) females. The present study was approved by the Institutional Review Board of The Affiliated Hospital of Nantong University, and all participants or their guardians provided written informed consent upon enrollment.

Baseline data collection. Baseline data were collected after consent for participation was gained, which included age, sex, immunoglobulin subtype, bone lesion, values for hemoglobin, calcium, serum creatinine, albumin, $\beta$-2-microglobulin $(\beta 2-\mathrm{MG})$, lactate dehydrogenase, Durie-Salmon stage (13), International Staging System (ISS) stage (14) and cytogenetic abnormalities. Cytogenetics abnormalities were previously determined at the hospital using fluorescence in situ hybridization, and the Durie-Salmon stage was evaluated according to the Durie-Salmon Criteria (classified as stage I-III).
Samples collection, processing and lnc-TCF7 detection. Bone marrow samples were extracted from patients with MM before treatment initiation, and from healthy donors after informed consent was obtained. Density gradient centrifugation (400 x g for $30 \mathrm{~min}$ at room temperature) was then immediately performed to separate the mononuclear cells from the samples, and the plasma cells were isolated using CD138-coated magnetic beads (Miltenyi Biotec $\mathrm{GmbH}$ ) according to the manufacturer's instructions. The levels of plasma cell lnc-TCF7 were detected using reverse transcription-quantitative (RT-q)PCR.

Follow up. The classification of MM was based on their immunoglobulin subtype, which was in accordance with the National Comprehensive Cancer Network Clinical Practice Guidelines in Oncology: Multiple Myeloma (15). All patients received the appropriate treatment according to these guidelines, based on their clinical status and willingness to receive treatment. Treatment included bortezomib/dexamethasone (BD), bortezomib/doxorubicin/dexamethasone (BDD) or melphalan/prednisone/bortezomib (MPB). Surveillance and follow up were performed every 3-6 months, or as indicated, which was performed in an out-patient clinic. The last follow-up date was June 2018, and the median follow-up duration was 29.0 months (range, 3.0 to 43.0 months). Complete response (CR), very good partial response (VGPR) and partial response (PR) were assessed based on the Revised Uniform Response Criteria by the International Myeloma Working Group (16). Overall response rate (ORR) was calculated as $\mathrm{ORR}=\mathrm{CR}+\mathrm{VGPR}+\mathrm{PR}$, and event-free survival time (EFS) was calculated from the date of treatment initiation to the date of disease relapse, progression or death. Overall survival time (OS) was calculated from the date of treatment initiation to the date of death.

Detection of lnc-TCF7 expression in MM cell lines. Human MM cell lines [OPM2, Roswell Park Memorial Institute (RPMI)8226, NCI-H929 and U266] were purchased from the Leibniz Institute DSMZ-German Collection of Microorganisms and Cell Cultures or The American Type Culture Collection, and cultured in 90\% RPMI-1640 medium supplemented with $10 \%$ fetal bovine serum (both Gibco; Thermo Fisher Scientific, Inc.). Subsequently, RT-qPCR was used to detect lnc-TCF7 expression in these cell lines, and in plasma cells isolated from BMMCs of health donors (as the control).

Plasmid transfection. Empty vector negative control (NC) and lnc-TCF7 short harpin (sh)RNA (Sh-TCF7) vectors were constructed by the Shanghai QeeJen Bio-tech Company. The vectors $(1 \mu \mathrm{g})$ were transfected into RPMI8226 cells with Lipofectamine 2000 (Invitrogen; Thermo Fisher Scientific, Inc.) for $24 \mathrm{~h}$ at $37^{\circ} \mathrm{C}$. After $48 \mathrm{~h}$ of transfection, the subsequent experiments were performed. Transfection efficiency was confirmed $24 \mathrm{~h}$ later using RT-qPCR.

Evaluation of cellular proliferation, apoptosis and potential target miRNAs following lnc-TCF7-knockdown. After plasmid transfection, cellular proliferation was detected at 0, 24, 48 and $72 \mathrm{~h}$ using a Cell Counting Kit-8 assay (Dojindo Molecular Technologies, Inc.) according to the manufacturer's instructions. Apoptosis rate was detected at $48 \mathrm{~h}$ using the Annexin V-FITC Apoptosis Detection kit (BD Biosciences) 
Table I. Primers used for quantitative PCR.

\begin{tabular}{lll}
\hline Gene & \multicolumn{1}{c}{ Forward primer, $5^{\prime} \rightarrow 3^{\prime}$} & \multicolumn{1}{c}{ Reverse primer, $5^{\prime} \rightarrow 3^{\prime}$} \\
\hline lnc-TCF7 & AATTTTGCACATCTGAACAGCC & TTCAAAATCCCACTACGCCCA \\
Jagged 1 & GGCTTGGATCTGTTGCTTGGT & TTGTTGGTGGTGTTGTCCTCAG \\
Notch1 & GCAACAGCGAGGAAGAGGAG & CGGCATCAGAGCGTGAGTAG \\
GAPDH & GACCACAGTCCATGCCATCAC & ACGCCTGCTTCACCACCTT \\
miR-203 & ACACTCCAGCTGGGAGTGGTTCTTAACAGT & TGTCGTGGAGTCGGCAATTC \\
miR-26b & ACACTCCAGCTGGGTTCAAGTAATTCAGGA & TGTCGTGGAGTCGGCAATTC \\
miR-643 & ACACTCCAGCTGGGACTTGTATGCTAGCTC & TGTCGTGGAGTCGGCAATTC \\
U6 & CGCTTCGGCAGCACATATACTA & ATGGAACGCTTCACGAATTTGC
\end{tabular}

miR, microRNA; lnc, long non-coding.

and a BD FACSCanto II flow cytometry (BD Biosciences) per the manufacturer's protocol. The analysis of apoptosis rate was performed by the FlowJo software (version 10; BD Biosciences). Furthermore, potential targets of 1nc-TCF7 were predicted using miRanda database analysis (www.miranda.org), and microRNA (miR)-203, miR-26b and miR-643 were selected for RT-qPCR validation and detection $24 \mathrm{~h}$ post-transfection.

Compensation experiments. Compensation experiments were performed to determine whether lnc-TCF7 shRNA influenced MM cells by targeting miR-203. RPMI8226 cells were transfected with miR-203 inhibitor negative control or miR-203 inhibitor, and the transfection efficacy was evaluated. Subsequently, RPMI8226 cells were transfected with empty vector plus miR-203 inhibitor NC (NC group), lnc-TCF7 shRNA alone (Sh-TCF7 group) or lnc-TCF7 shRNA combined with an miR-203 inhibitor (Sh-TCF7/miR inhibitor group). The expression levels of lnc-TCF7 and miR-203 were detected by RT-qPCR $24 \mathrm{~h}$ later. Cellular proliferation and apoptosis analysis were conducted as aforementioned.

$R T$ - $q P C R$. Total RNA was extracted from RPMI8226 cells using TRIzol ${ }^{\circledR}$ reagent (Invitrogen; Thermo Fisher Scientific, Inc.) according to the manufacturer's instructions. The extracted RNA was then reverse transcribed into cDNA using the PrimeScript RT reagent kit (Takara Biotechnology, Co., Ltd.) at $42^{\circ} \mathrm{C}$ for $2 \mathrm{~min}$, and qPCR was subsequently performed with the ABI 7500 system (Applied Biosystems; Thermo Fisher Scientific, Inc.) using the SYBR Premix Ex Taq kit (Takara Biotechnology, Co., Ltd.) $\left(95^{\circ} \mathrm{C}\right.$ for $30 \mathrm{sec}$; 40 cycles of $95^{\circ} \mathrm{C}$ for $5 \mathrm{sec}$ and $60^{\circ} \mathrm{C}$ for $10 \mathrm{sec}$ ). GAPDH was used as the internal reference for IncRNA and mRNA, while U6 was used as internal reference to miRNA expression analysis. Relative RNA expression was calculated using the $2^{-\Delta \Delta \mathrm{Cq}}$ method (17). The qPCR primer sequences are listed in Table I.

Western blotting. Total protein was isolated from cells using RIPA Lysis and Extraction Buffer (Thermo Fisher Scientific, Inc.), and the protein concentration was determined using the Pierce $^{\mathrm{TM}}$ BCA Protein Assay kit (Thermo Fisher Scientific, Inc.). The protein samples (20 $\mu \mathrm{g}$ per lane) were then separated by $4-20 \%$ SDS-PAGE and transferred to polyvinylidene fluoride membranes (EMD Millipore). After blocking with 5\% non-fat milk for $2 \mathrm{~h}$ at $37^{\circ} \mathrm{C}$, the membranes were incubated with the corresponding primary antibodies overnight at $4^{\circ} \mathrm{C}$. The membranes were subsequently incubated with secondary antibody for $1 \mathrm{~h}$ at room temperature. The bands were visualized using Pierce ${ }^{\mathrm{TM}}$ ECL Plus Western Blotting Substrate (Thermo Fisher Scientific, Inc.) followed by exposure to X-ray film (Kodak). The greyscales of the blots were analyzed using ImageJ software (ver.1.8; National Institutes of Health). All antibodies are listed in Table II.

Statistical analysis. The study data are presented as the mean \pm standard deviation, median and interquartile range or count (percentage), and statistical analysis was performed using SPSS 24.0 (IBM Corp). Comparisons among three or more groups were made using the Kruskal Wallis test, or one-way ANOVA followed by Dunnett's multiple comparison test or Tukey's multiple comparison test. Comparisons between two groups were made using the unpaired t-test, Wilcoxon rank sum test or the $\chi^{2}$ test. Univariate and multivariate logistic regression analyses were performed to screen factors affecting CR. Univariate and multivariate Cox proportional hazards regression model analyses were performed to screen factors affecting EFS and OS. All graphs were plotted using GraphPad Prism 7.01 (GraphPad Software, Inc.). Receiver operating curve (ROC) analysis and area under the curve (AUC) were conducted to assess the diagnostic value of lnc-TCF7 in MM. OS and EFS are displayed as Kaplan-Meier curves. Patients with MM were divided into lnc-TCF7-high and -low expression groups based on the median lnc-TCF7 level (1.91), and the survival difference between the high- and low-lnc-TCF7 expression groups was determined using the log-rank test. $\mathrm{P}<0.05$ was considered to indicate a statistically significant difference.

\section{Results}

Baseline characteristics of patients with MM. Our preliminary research (with a small sample population) indicated that lnc-TCF7 was upregulated in patients with MM compared with healthy controls, and in MM cells compared with BMMCs plasma cells from health donors (data not shown). Of the 86 patients with MM, the mean age was $58.9 \pm 9.0$ years, including $53(61.6 \%)$ men and $33(38.4 \%)$ women. For Ig subtype, the numbers of patients positive for $\operatorname{IgG}, \operatorname{IgA}, \operatorname{IgM}$, 
Table II. Antibodies using in western blotting.

A, Primary antibodies

\begin{tabular}{cccc}
\hline Antibody & Company & Catalog number & Dilution \\
Jagged1 Rabbit mAb & CST & $\# 2620$ & $1: 1,000$ \\
Notch1 Rabbit mAb & CST & $\# 3268$ & $1: 1,000$ \\
GAPDH Rabbit mAb & CST & $\# 2118$ & $1: 1,000$ \\
\hline
\end{tabular}

$\mathrm{B}$, Secondary antibody

\begin{tabular}{cccc}
\hline Antibody & Company & Catalog number & Dilution \\
Goat Anti-Rabbit & CST & $\# 7074$ & $1: 3,000$ \\
IgG H\&L (HRP) & & & \\
\hline
\end{tabular}

CST, Cell Signaling Technology, Inc.; HRP, horseradish peroxidase.

IgD and free light chain were 49 (57.0\%), 20 (23.2\%), 1 (1.2\%), $1(1.2 \%)$ and 15 (17.4\%), respectively. For Durie-Salmon stage, the numbers of patient with stage I, II and III disease were $3(3.5 \%), 40(46.5 \%)$ and $43(50.0 \%)$, respectively, and the numbers at ISS stage I, II and III were 19 (22.1\%), 32 (37.2\%) and $35(40.7 \%)$, respectively. Details of the other baseline characteristics are displayed in Table III.

Relation between lnc-TCF7 expression and risk of MM. lnc-TCF7 expression was increased in patients with MM [median value of relative expression: 1.91 (0.98-3.75)] compared with healthy controls [median value of relative expression: 0.64 (0.30-1.01)] $(\mathrm{P}<0.001)$ (Fig. 1A). ROC curve analysis revealed that lnc-TCF7 effectively discriminated between patients and healthy controls, with an AUC of 0.832 (95\% confidence interval, 0.749-0.915; Fig. 1B), suggesting that lnc-TCF7 was related to increased risk of MM. No differences in lnc-TCF7 expression were observed among patients with MM who had received BD, BDD or MPB treatment (Fig. S1).

Relation between Inc-TCF7 expression and the clinicopathological characteristics of patients with MM. 1nc-TCF7 expression was found to be significantly related to $\beta 2-\mathrm{MG}$ expression $(\mathrm{P}=0.001)$ and ISS stage $(\mathrm{P}=0.001)$, though no difference was observed between Inc-TCF7 expression and other baseline clinical characteristics (Table IV).

Effects of lnc-TCF7 expression on treatment responses and survivals in patients with MM. $\mathrm{CR}(\mathrm{P}=0.079$; Fig. $2 \mathrm{~A})$ was lower, while ORR ( $\mathrm{P}=0.436$; Fig. $2 \mathrm{~B})$ was similar in the lnc-TCF7-high expression group compared with the low expression group. Furthermore, EFS ( $\mathrm{P}=0.047$; Fig. 2C) and OS ( $\mathrm{P}=0.004$; Fig. 2D) time were shorter in the lnc-TCF7-high compared with lnc-TCF7-low expression group, indicating that increased lnc-TCF7 expression was related to poorer prognosis of patients with MM.

Analyses of factors affecting $C R$. The results of univariate or multivariate logistic regression analysis revealed that lnc-TCF7 expression and any other clinical characteristics were not related to a $\mathrm{CR}$ in patients with $\mathrm{MM}$ (all $\mathrm{P}>0.05$; Table V).
Table III. Characteristics of 86 patients with multiple myeloma.

\begin{tabular}{|c|c|}
\hline Parameters & Value \\
\hline Age, years, mean \pm SD & $58.9 \pm 9.0$ \\
\hline \multicolumn{2}{|l|}{$\operatorname{Sex}, \mathrm{n}(\%)$} \\
\hline Male & $53(61.6)$ \\
\hline Female & $33(38.4)$ \\
\hline \multicolumn{2}{|l|}{ Immunoglobulin subtype, $\mathrm{n}(\%)$} \\
\hline $\operatorname{IgG}$ & $49(57.0)$ \\
\hline $\operatorname{Ig} \mathrm{A}$ & $20(23.2)$ \\
\hline $\operatorname{IgM}$ & $1(1.2)$ \\
\hline $\operatorname{IgD}$ & $1(1.2)$ \\
\hline Free light chain & $15(17.4)$ \\
\hline Bone lesion, $\mathrm{n}(\%)$ & $64(74.4)$ \\
\hline $\mathrm{Hb}, \mathrm{g} / \mathrm{dl}$, median (IQR) & $10.1(8.6-11.5)$ \\
\hline Calcium, mg/dl, median (IQR) & $9.9(8.9-11.4)$ \\
\hline Scr, mg/dl, median (IQR) & $1.6(1.3-1.9)$ \\
\hline ALB, mg/dl, median (IQR) & $3.9(3.3-4.2)$ \\
\hline ß2-MG, mg/l, median (IQR) & $4.7(2.8-8.9)$ \\
\hline LDH, U/1, median (IQR) & $193.0(167.8-228.7)$ \\
\hline \multicolumn{2}{|l|}{ Durie-Salmon stage, $\mathrm{n}(\%)$} \\
\hline I & $3(3.5)$ \\
\hline II & $40(46.5)$ \\
\hline III & $43(50.0)$ \\
\hline \multicolumn{2}{|l|}{ ISS stage, n (\%) } \\
\hline I & $19(22.1)$ \\
\hline II & $32(37.2)$ \\
\hline III & $35(40.7)$ \\
\hline \multicolumn{2}{|l|}{ Cytogenetics, n (\%) } \\
\hline $\mathrm{t}(4 ; 14)$ & $7(8.1)$ \\
\hline $\mathrm{t}(14 ; 16)$ & $11(12.8)$ \\
\hline $\operatorname{Del}(17 \mathrm{p})$ & $12(14.0)$ \\
\hline
\end{tabular}

$\mathrm{SD}$, standard deviation; Ig, immunoglobulin; IQR, interquartile range; $\mathrm{Hb}$, hemoglobin; Scr, serum creatinine; ALB, albumin; $\beta 2-\mathrm{MG}$, $\beta 2$-microglobulin; LDH, lactate dehydrogenase; ISS, international staging system; t, translocation; del, deletion.

Analyses offactors affecting EFS. Univariate Cox proportional hazards regression model analysis showed that lnc-TCF7 expression (high vs. low) was related to EFS, though this was not statistically significant $(\mathrm{P}=0.052)$. However, ISS stage (III vs. II/I) was significanlty related to shorter EFS $(\mathrm{P}<0.001$; Table VI). All factors were further analyzed using multivariate Cox analysis, which disclosed that lnc-TCF7 expression (high vs. low) was not an independent risk factor for $\mathrm{EFS}(\mathrm{P}=0.307)$; whereas age ( $>60$ vs. $\leq 60$ years; $\mathrm{P}=0.029)$, Durie-Salmon stage (III vs. II/I; P=0.012) and ISS stage (III vs. II/I; P<0.001) were independent risk factors for shorter EFS; however, age and Durie-Salmon stage were non-significant in the univariate analysis (Table VI).

Analyses of factors affecting OS. In order to further investigate the factors affecting OS in patients with MM, univariate Cox proportional hazards regression model analysis was 

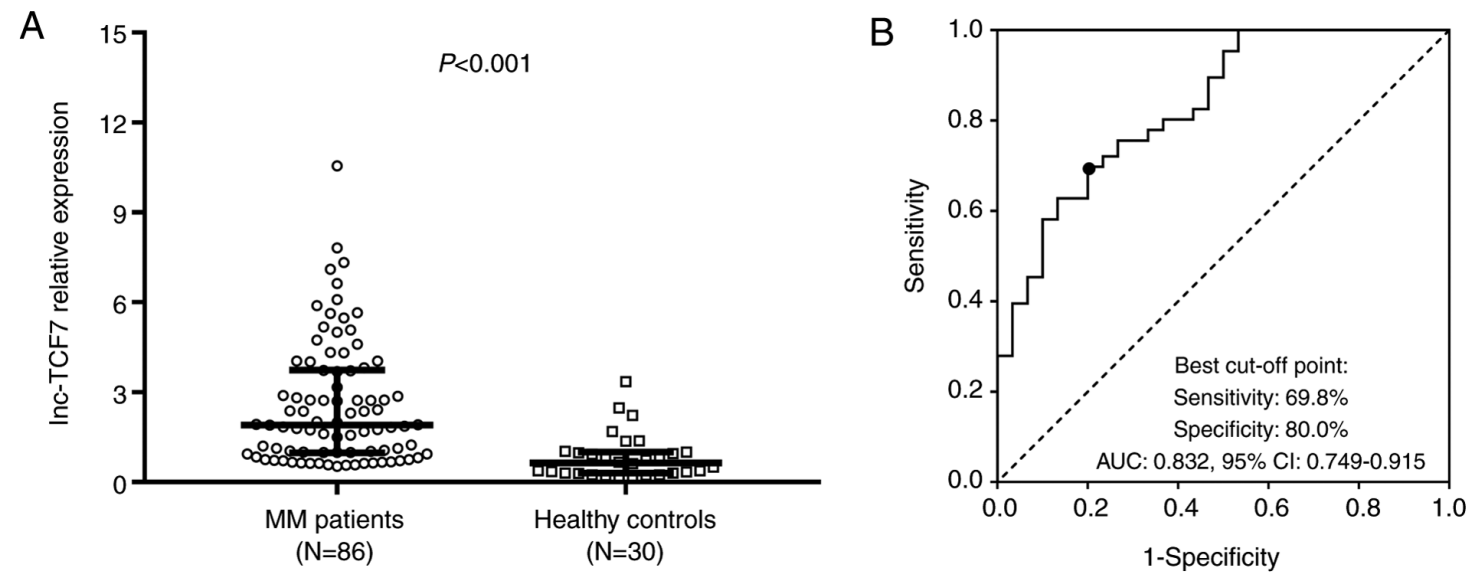

Figure 1. Comparison of 1nc-TCF7 expression levels between patients with MM and healthy controls. (A) lnc-TCF7 expression was elevated in patients with MM compared with healthy controls, (B) and is an effective predictor of MM risk. lnc-TCF7, long non-coding RNA transcription factor 7; MM, multiple myeloma; ROC, receiver operating characteristic; AUC, area under the curve.


Figure 2. Relation between lnc-TCF7 expression and prognosis in patients with MM. (A) CR was numerically lower (but without statistical significance) while (B) ORR was similar in the lnc-TCF7-high expression group compared with the low expression group. (C) EFS and (D) OS were decreased in the lnc-TCF7-high group compared with the lnc-TCF7-low group. lnc-TCF7, long non-coding RNA transcription factor 7; MM, multiple myeloma; CR, complete response; ORR, overall response rate; EFS, event-free survival; OS, overall survival.

performed, which revealed that lnc-TCF7 expression (high vs. low; $\mathrm{P}=0.008$ ) and ISS stage (III vs. II/I; P<0.001) were related to worse OS (Table VII). All factors were further subjected to multivariate Cox analysis, which showed that lnc-TCF7 expression (high vs. low; $\mathrm{P}=0.123$ ) was not an independent factor for predicting OS, while ISS stage (III vs. II/I; P<0.001) was an independent predictive factor for poorer OS (Table VII). These results suggested that lnc-TCF7 may influence ISS stage rather than directly impacting OS in patients with MM.

Comparison of lnc-TCF7 expression between MM cell lines and plasma cells from healthy BMMCs. The expression levels of 1nc-TCF7 were subsequently compared between MM cell lines and BMMC from healthy donors. The results revealed 
Table IV. Relation between lnc-TCF7 relative expression and clinical characteristics.

\begin{tabular}{|c|c|c|}
\hline Variables & $\begin{array}{c}\text { lnc-TCF7 relative } \\
\text { expression, median } \\
\text { (IQR) }\end{array}$ & P-value \\
\hline Age, years & & 0.242 \\
\hline$\leq 60$ & $2.33(0.95-4.11)$ & \\
\hline$>60$ & $1.59(1.00-3.07)$ & \\
\hline Sex & & 0.912 \\
\hline Female & $1.94(1.03-3.03)$ & \\
\hline Male & $1.90(0.89-4.03)$ & \\
\hline Immunoglobulin subtype & & 0.209 \\
\hline $\operatorname{IgG}$ & $1.84(0.83-3.92)$ & \\
\hline IgA & $1.72(0.96-2.41)$ & \\
\hline $\operatorname{IgM}$ & $1.99^{\mathrm{a}}$ & \\
\hline $\mathrm{IgD}$ & $7.11^{\mathrm{a}}$ & \\
\hline Free light chain & $2.74(1.24-5.08)$ & \\
\hline Bone lesion & & 0.138 \\
\hline No & $2.55(1.10-5.25)$ & \\
\hline Yes & $1.81(0.86-3.10)$ & \\
\hline $\mathrm{Hb}, \mathrm{g} / \mathrm{dl}$ & & 0.349 \\
\hline$\leq 10$ & $1.87(1.07-4.61)$ & \\
\hline$>10$ & $2.02(0.84-2.81)$ & \\
\hline Calcium, mg/dl & & 0.292 \\
\hline$\leq 11.5$ & $1.85(0.92-2.96)$ & \\
\hline$>11.5$ & $2.57(1.00-4.64)$ & \\
\hline Scr, mg/dl & & 0.355 \\
\hline$\leq 2$ & $1.90(0.94-3.17)$ & \\
\hline$>2$ & $3.73(1.07-5.18)$ & \\
\hline $\mathrm{ALB}, \mathrm{mg} / \mathrm{dl}$ & & 0.878 \\
\hline$\leq 3.5$ & $1.96(1.00-3.39)$ & \\
\hline$>3.5$ & $1.85(0.86-3.79)$ & \\
\hline$\beta 2-\mathrm{MG}, \mathrm{mg} / \mathrm{l}$ & & 0.001 \\
\hline$\leq 5.5$ & $1.57(0.74-2.30)$ & \\
\hline$>5.5$ & $2.87(1.74-4.34)$ & \\
\hline $\mathrm{LDH}, \mathrm{U} / \mathrm{l}$ & & 0.763 \\
\hline$\leq 220$ & $1.85(0.96-3.96)$ & \\
\hline$>220$ & $2.19(0.98-3.75)$ & \\
\hline Durie-Salmon stage & & 0.297 \\
\hline I & $0.75(0.67-2.24)$ & \\
\hline II & $2.36(0.84-4.02)$ & \\
\hline III & $1.83(1.07-3.72)$ & \\
\hline ISS stage & & 0.001 \\
\hline I & $1.24(0.74-1.83)$ & \\
\hline II & $1.81(0.73-4.24)$ & \\
\hline III & $2.87(1.74-4.34)$ & \\
\hline $\mathrm{t}(4 ; 14)$ & & 0.076 \\
\hline No & $1.87(0.94-3.72)$ & \\
\hline Yes & $2.73(1.79-7.33)$ & \\
\hline $\mathrm{t}(14 ; 16)$ & & 0.693 \\
\hline No & $1.94(0.84-3.73)$ & \\
\hline Yes & $1.51(1.07-5.89)$ & \\
\hline
\end{tabular}

Table IV. Continued.

\begin{tabular}{lcc}
\hline Variables & $\begin{array}{c}\text { Inc-TCF7 relative } \\
\text { expression, median } \\
\text { (IQR) }\end{array}$ & P-value \\
\hline Del(17p) & & 0.236 \\
No & $1.85(0.92-3.70)$ & \\
Yes & $2.81(1.15-4.76)$ & \\
\hline
\end{tabular}

${ }^{\text {aData }}$ were only available for one patient, therefore there are no IQRs. Comparisons were determined by Wilcoxon rank sum test or Kruskal Wallis test. IQR, interquartile range; Ig, immunoglobulin; $\mathrm{Hb}$, hemoglobin; Scr, serum creatinine; ALB, albumin; $\beta 2-\mathrm{MG}$, $\beta 2$-microglobulin; LDH, lactate dehydrogenase; ISS, international staging system; t, translocation; del, deletion.

that lnc-TCF7 expression was elevated in MM cell lines [OPM2 $(\mathrm{P}<0.05)$, RPMI8226 ( $\mathrm{P}<0.001)$, NCI-H929 $(\mathrm{P}<0.01)$ and $\mathrm{U} 266$ $(\mathrm{P}<0.001)]$ compared with healthy donor cells (Fig. 3).

Effects of Inc-TCF7-knockdown on cellular proliferation and apoptosis, and its potential target miRNAs in RPMI8226 cells. The transfection efficacy was evaluated by RT-qPCR, which is presented in Fig. S2. Following transfection, lnc-TCF7 expression was decreased in the Sh-TCF7 group compared with the NC group, suggesting that the transfection of the lnc-TCF7 shRNA vector into RPMI8226 cells was successful ( $\mathrm{P}<0.001$; Fig. 4A). RPMI8226 cell proliferation was inhibited at $48(\mathrm{P}<0.05)$ and $72 \mathrm{~h}(\mathrm{P}<0.05)($ Fig. $4 \mathrm{~B})$ post-transfection, while apoptosis $(\mathrm{P}<0.01)$ (Fig. $4 \mathrm{C}$ and $\mathrm{D})$ was enhanced in the Sh-TCF7 group compared with the NC group, indicating that lnc-TCF7-knockdown suppressed cellular proliferation while promoting apoptosis in MM cells. In addition, potential targets of Inc-TCF7 were predicted using the miRanda database, and miR-203, $-26 \mathrm{~b}$ and -643 were selected for validation and detection. The results indicated that miR-203 was upregulated in the Sh-TCF7 group compared with the NC group $(\mathrm{P}<0.01$; Fig. 4E), whereas miR-26b ( $\mathrm{P}>0.05$; Fig. 4F) and miR-643 ( $\mathrm{P}>0.05$; Fig. 4G) levels were similar between the two groups, illustrating that lnc-TCF7-knockdown upregulated miR-203 expression but did not affect that of miR-26b or -643 in RPMI8226 cells.

Interaction between Inc-TCF7 and miR-203 mediated the Jagged1-Notch1 signaling pathway in RPMI8226 cells. Compensation experiments were performed to determine whether lnc-TCF7 shRNA functions in MM cells by enhancing miR-203 and regulating the downstream Jagged1-Notch1 signaling pathway. The results indicated that miR-203 expression was decreased in the Sh-TCF $7 / \mathrm{miR}$ inhibitor group compared with Sh-TCF7 group $(\mathrm{P}<0.01$; Fig. 5A), whereas lnc-TCF7 expression remained consistent between the two groups ( $\mathrm{P}>0.05$; Fig. $5 \mathrm{~B}$ ), indicating that lnc-TCF7 suppressed miR-203 expression, but miR-203 did not influence lnc-TCF7 expression. In addition, cellular proliferation was enhanced at $72 \mathrm{~h}(\mathrm{P}<0.05$; Fig. $5 \mathrm{C})$, while apoptosis was repressed $(\mathrm{P}<0.05)$ (Fig. 5D and E) in the Sh-TCF7/miR inhibitor compared with the Sh-TCF7 groups. These findings suggested that miR-203 inhibition accelerated cellular proliferation while reducing 
Table V. Univariate and multivariate logistic regression model analyses of factors affecting complete response.

\begin{tabular}{|c|c|c|c|c|}
\hline \multirow[b]{2}{*}{ Variables } & \multicolumn{2}{|c|}{ Univariate logistic regression } & \multicolumn{2}{|c|}{ Multivariate logistic regression } \\
\hline & P-value & OR $(95 \% \mathrm{CI})$ & P-value & OR $(95 \% \mathrm{CI})$ \\
\hline lnc-TCF7, high vs. low & 0.084 & $0.403(0.144-1.129)$ & 0.398 & $0.585(0.169-2.028)$ \\
\hline Age, $>60$ years vs. $\leq 60$ years & 0.923 & $1.051(0.381-2.900)$ & 0.680 & $0.767(0.217-2.712)$ \\
\hline Sex, male vs. female & 0.627 & $0.780(0.287-2.122)$ & 0.352 & $0.536(0.144-1.992)$ \\
\hline Immunoglobulin subtype, IgG vs. others & 0.986 & $1.009(0.373-2.726)$ & 0.836 & $1.134(0.345-3.725)$ \\
\hline Bone lesion, yes vs. no & 0.718 & $0.816(0.271-2.458)$ & 0.892 & $0.901(0.200-4.069)$ \\
\hline $\mathrm{Hb},>10 \mathrm{~g} / \mathrm{dl}$ vs. $\leq 10 \mathrm{~g} / \mathrm{dl}$ & 0.084 & $0.403(0.144-1.129)$ & 0.262 & $0.412(0.087-1.941)$ \\
\hline Calcium, $>11.5 \mathrm{mg} / \mathrm{dl}$ vs. $\leq 11.5 \mathrm{mg} / \mathrm{dl}$ & 0.601 & $0.721(0.211-2.457)$ & 0.871 & $0.881(0.190-4.075)$ \\
\hline $\mathrm{Scr},>2.0 \mathrm{mg} / \mathrm{dl} \mathrm{vs} . \leq 2.0 \mathrm{mg} / \mathrm{dl}$ & 0.330 & $1.950(0.509-7.461)$ & 0.186 & $3.238(0.567-18.483)$ \\
\hline ALB,$>3.5 \mathrm{mg} / \mathrm{dl} \mathrm{vs.} \leq 3.5 \mathrm{mg} / \mathrm{dl}$ & 0.486 & $1.463(0.501-4.277)$ & 0.590 & $1.405(0.407-4.850)$ \\
\hline $\mathrm{LDH},>220 \mathrm{U} / 1 \mathrm{vs} . \leq 220 \mathrm{U} / 1$ & 0.463 & $0.655(0.211-2.028)$ & 0.820 & $0.859(0.232-3.180)$ \\
\hline Durie-Salmon stage, III vs. II/I & 0.213 & $1.896(0.693-5.188)$ & 0.967 & $0.968(0.202-4.630)$ \\
\hline ISS stage, III vs. II/I & 0.076 & $0.365(0.119-1.113)$ & 0.163 & $0.328(0.068-1.570)$ \\
\hline $\mathrm{t}(4 ; 14)$, yes vs. no & 0.523 & $0.492(0.056-4.336)$ & 0.945 & $0.913(0.067-12.354)$ \\
\hline $\mathrm{t}(14 ; 16)$, yes vs. no & 0.608 & $0.655(0.130-3.303)$ & 0.717 & $0.691(0.094-5.101)$ \\
\hline $\operatorname{Del}(17 p)$, yes vs. no & 0.192 & $0.245(0.030-2.025)$ & 0.235 & $0.228(0.020-2.619)$ \\
\hline
\end{tabular}

OR, odds ratio; CI, confidence interval; Ig, immunoglobulin; Hb, hemoglobin; Scr, serum creatinine; ALB, albumin; LDH, lactate dehydrogenase; ISS, international staging system; t, translocation; del, Deletion.

Table VI. Univariate and multivariate Cox's proportional hazards regression model analyses of factors affecting event-free survival.

\begin{tabular}{|c|c|c|c|c|}
\hline \multirow[b]{2}{*}{ Variables } & \multicolumn{2}{|c|}{ Univariate Cox's regression } & \multicolumn{2}{|c|}{ Multivariate Cox's regression } \\
\hline & P-value & HR $(95 \% \mathrm{CI})$ & P-value & $\mathrm{HR}(95 \% \mathrm{CI})$ \\
\hline lnc-TCF7, high vs. low & 0.052 & $1.878(0.994-3.549)$ & 0.307 & $1.535(0.674-3.495)$ \\
\hline Age, $>60$ years vs. $\leq 60$ years & 0.564 & $1.203(0.642-2.254)$ & 0.029 & $2.561(1.102-5.954)$ \\
\hline Sex, male vs. female & 0.111 & $0.602(0.322-1.124)$ & 0.846 & $1.086(0.472-2.503)$ \\
\hline $\begin{array}{l}\text { Immunoglobulin subtype, } \\
\text { IgG vs. others }\end{array}$ & 0.796 & $1.087(0.577-2.047)$ & 0.781 & $0.899(0.424-1.907)$ \\
\hline Bone lesion, yes vs. no & 0.171 & $0.622(0.315-1.228)$ & 0.208 & $0.547(0.214-1.400)$ \\
\hline $\mathrm{Hb},>10 \mathrm{~g} / \mathrm{dl}$ vs. $\leq 10 \mathrm{~g} / \mathrm{dl}$ & 0.614 & $0.852(0.457-1.588)$ & 0.754 & $1.153(0.474-2.804)$ \\
\hline Calcium, $>11.5 \mathrm{mg} / \mathrm{dl}$ vs. $\leq 11.5 \mathrm{mg} / \mathrm{dl}$ & 0.492 & $1.287(0.627-2.640)$ & 0.922 & $0.955(0.379-2.408)$ \\
\hline $\mathrm{Scr},>2.0 \mathrm{mg} / \mathrm{dl} \mathrm{vs} . \leq 2.0 \mathrm{mg} / \mathrm{dl}$ & 0.260 & $1.653(0.690-3.959)$ & 0.339 & $0.575(0.185-1.790)$ \\
\hline ALB,$>3.5 \mathrm{mg} / \mathrm{dl}$ vs. $\leq 3.5 \mathrm{mg} / \mathrm{dl}$ & 0.643 & $1.170(0.603-2.269)$ & 0.602 & $1.234(0.560-2.718)$ \\
\hline LDH, >220 U/1 vs. $\leq 220 \mathrm{U} / 1$ & 0.316 & $1.397(0.727-2.684)$ & 0.151 & $1.850(0.799-4.283)$ \\
\hline Durie-Salmon stage, III vs. II/I & 0.142 & $1.608(0.852-3.034)$ & 0.012 & $3.230(1.295-8.055)$ \\
\hline ISS stage, III vs. II/I & $1.2 \times 10-7$ & $6.919(3.383-14.153)$ & $1.9 \times 10-7$ & $12.746(4.892-33.208)$ \\
\hline $\mathrm{t}(4 ; 14)$, yes vs. no & 0.135 & $2.051(0.800-5.254)$ & 0.996 & $0.997(0.304-3.263)$ \\
\hline $\mathrm{t}(14 ; 16)$, yes vs. no & 0.689 & $1.194(0.501-2.848)$ & 0.977 & $0.986(0.368-2.639)$ \\
\hline $\operatorname{Del}(17 p)$, yes vs. no & 0.152 & $1.763(0.811-3.832)$ & 0.655 & $0.792(0.284-2.206)$ \\
\hline
\end{tabular}

HR, hazard ratio; CI, confidence interval; Ig, immunoglobulin; Hb, hemoglobin; Scr, serum creatinine; ALB, albumin; LDH, lactate dehydrogenase; ISS, international staging system; t, translocation; Del, deletion.

inhibition in lnc-TCF7-knockdown cells. Furthermore, miRanda database analysis predicted Jagged1 to be a target gene of miR-203, and the Jagged1-Notch1 signaling pathway has been reported to be directly regulated by miR-203 (18). Thus, in the present study, Jagged1 and Notch1 expression were detected using western blotting and RT-qPCR. Jagged1 
Table VII. Univariate and multivariate Cox's proportional hazards regression model analyses of factors affecting overall survival.

\begin{tabular}{|c|c|c|c|c|}
\hline \multirow[b]{2}{*}{ Variables } & \multicolumn{2}{|c|}{ Univariate Cox regression } & \multicolumn{2}{|c|}{ Multivariate Cox regression } \\
\hline & P-value & $\mathrm{HR}(95 \% \mathrm{CI})$ & P-value & $\mathrm{HR}(95 \% \mathrm{CI})$ \\
\hline lnc-TCF7, high vs. low & 0.008 & $3.513(1.391-8.874)$ & 0.123 & $2.541(0.778-8.302)$ \\
\hline Age, $>60$ years vs. $\leq 60$ years & 0.874 & $1.068(0.474-2.407)$ & 0.233 & $1.991(0.642-6.178)$ \\
\hline Sex, male vs. female & 0.089 & $0.498(0.223-1.111)$ & 0.471 & $0.648(0.199-2.109)$ \\
\hline $\begin{array}{l}\text { Immunoglobulin subtype, } \\
\text { IgG vs. others }\end{array}$ & 0.724 & $0.865(0.387-1.934)$ & 0.705 & $0.832(0.320-2.162)$ \\
\hline Bone lesion, yes vs. no & 0.942 & $0.964(0.357-2.602)$ & 0.657 & $0.705(0.150-3.306)$ \\
\hline $\mathrm{Hb},>10 \mathrm{~g} / \mathrm{dl}$ vs. $\leq 10 \mathrm{~g} / \mathrm{dl}$ & 0.751 & $1.139(0.509-2.548)$ & 0.952 & $0.959(0.249-3.699)$ \\
\hline $\begin{array}{l}\text { Calcium, }>11.5 \mathrm{mg} / \mathrm{dl} \text { vs. } \\
\leq 11.5 \mathrm{mg} / \mathrm{dl}\end{array}$ & 0.105 & $2.033(0.863-4.790)$ & 0.225 & $2.301(0.599-8.841)$ \\
\hline $\mathrm{Scr},>2.0 \mathrm{mg} / \mathrm{dl} \mathrm{vs} . \leq 2.0 \mathrm{mg} / \mathrm{dl}$ & 0.939 & $1.058(0.246-4.544)$ & 0.616 & $0.612(0.090-4.170)$ \\
\hline ALB,$>3.5 \mathrm{mg} / \mathrm{dl}$ vs. $\leq 3.5 \mathrm{mg} / \mathrm{dl}$ & 0.833 & $1.096(0.468-2.562)$ & 0.227 & $2.010(0.647-6.242)$ \\
\hline LDH, $>220 \mathrm{U} / 1$ vs. $\leq 220 \mathrm{U} / 1$ & 0.172 & $1.763(0.782-3.975)$ & 0.097 & $2.634(0.839-8.266)$ \\
\hline Durie-Salmon stage, III vs. II/I & 0.516 & $1.309(0.581-2.952)$ & 0.316 & $1.921(0.536-6.878)$ \\
\hline ISS stage, III vs. II/I & $7.7 \times 10-7$ & $11.113(4.276-28.882)$ & $3.4 \times 10-5$ & $17.494(4.519-67.727)$ \\
\hline $\mathrm{t}(4 ; 14)$, yes vs. no & 0.110 & $2.408(0.818-7.086)$ & 0.699 & $0.725(0.142-3.710)$ \\
\hline $\mathrm{t}(14 ; 16)$, yes vs. no & 0.471 & $0.587(0.138-2.500)$ & 0.490 & $0.563(0.110-2.875)$ \\
\hline $\operatorname{Del}(17 p)$, yes vs. no & 0.618 & $1.315(0.449-3.854)$ & 0.299 & $0.446(0.097-2.047)$ \\
\hline
\end{tabular}

OS, overall survival; HR, hazard ratio; CI, confidence interval; Ig, immunoglobulin; Hb, hemoglobin; Scr, serum creatinine; ALB, albumin; LDH, lactate dehydrogenase; ISS, international staging system; t, translocation; Del, deletion.

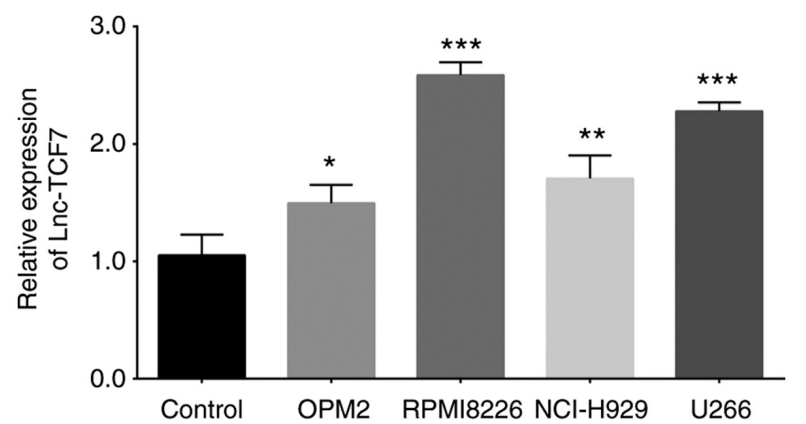

Figure 3. Comparison of lnc-TCF7 level between MM cell lines and BMMC plasma cells from healthly donors. lnc-TCF7 levels in MM cell lines (OPM2, RPMI8226, NCI-H929 and U266) were higher compared with those in plasma cells from healthy BMMCs. ${ }^{*} \mathrm{P}<0.05,{ }^{* *} \mathrm{P}<0.01,{ }^{* * * *} \mathrm{P}<0.001$. Inc-TCF7, long non-coding RNA transcription factor 7; MM, multiple myeloma; BMMCs, bone marrow mononuclear cells.

$(\mathrm{P}<0.05)$ (Fig. 5F and $\mathrm{G})$ and Notch1 $(\mathrm{P}<0.05)($ Fig. 5F and H) expression were detected using western blot and RT-qPCR analyses, which revealed that both were upregulated in the Sh-TCF7/miR inhibitor group compared with the Sh-TCF7 group. These data indicated that lnc-TCF7-knockdown suppressed cellular proliferation while promoting apoptosis by regulating the miR-203-mediated Jagged1-Notch1 signaling pathway in RPMI8226 cells.

\section{Discussion}

lnc-TCF7, a newly identified lncRNA, has been reported to be upregulated in various types of cancer (8-10). For example,
Wang et al (7) reported that, in 39 patients with liver cancer, lnc-TCF7 was upregulated in tumor tissues compared with paired-adjacent normal tissues. Furthermore, Li et al (10) revealed that lnc-TCF7 expression was increased in tumor tissues compared with adjacent normal tissues in patients with CRC, and that increased lnc-TCF7 levels were associated with larger tumors and a higher Tumor-Node-Metastasis stage. These studies reveal that lnc-TCF7 expression is elevated in several types of solid tumor, and is associated with deteriorating clinical features. Another previous study demonstrated that the associated lnc-TCF7 gene (TCF7) regulates the functions of malignant hematological cells (11). Our preliminary research (with a small sample population) indicated that lnc-TCF7 was upregulated in patients with MM compared with healthy controls, and in MM cells compared with BMMCs plasma cells from health donors (unpublished data). Based on these findings, it was hypothesized that lnc-TCF7 may also be upregulated in patients with MM, which may be used to predict the clinical features of MM progression. However, limited information could be obtained. In the current study, lnc-TCF7 expression was found to be elevated in patients with MM compared with healthy controls, which effectively predicted an increased risk of MM. In addition, lnc-TCF7 expression was related to $\beta 2-\mathrm{MG}$ level and the ISS stage. There are a number of possible reasons for these results. Firstly, lnc-TCF7 may act as a competing endogenous RNA (ceRNA) that sponges specific tumor suppressor genes (including miR-203) and stimulates MM tumorigenesis. This potentially increases the risk of $\mathrm{MM}$, as increased $\beta 2-\mathrm{MG}$ level may be related to more advanced ISS stage. Secondly, lncRNAs may directly activate specific signaling pathways 
A

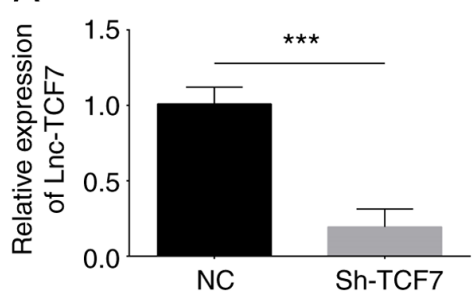

B

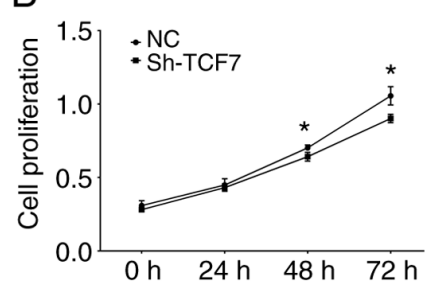

C
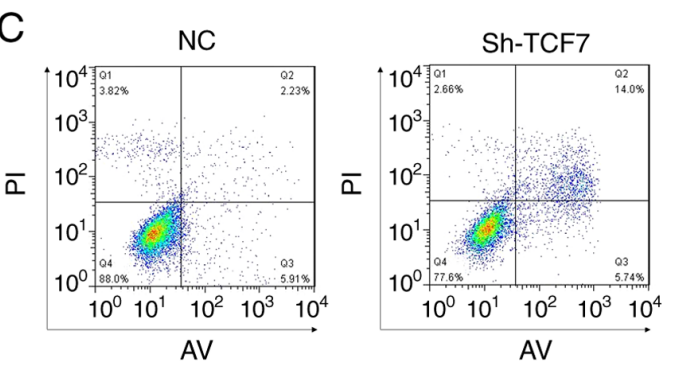

$\mathrm{D}$

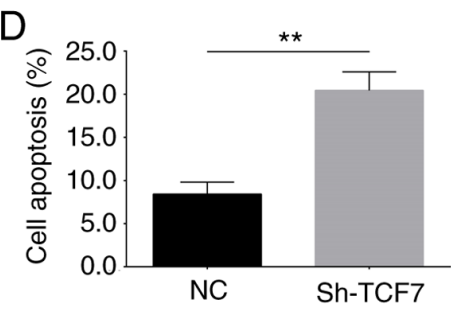

$\mathrm{F}$

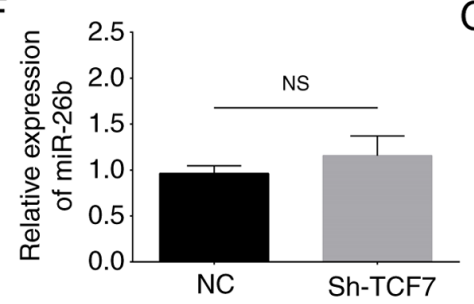

E

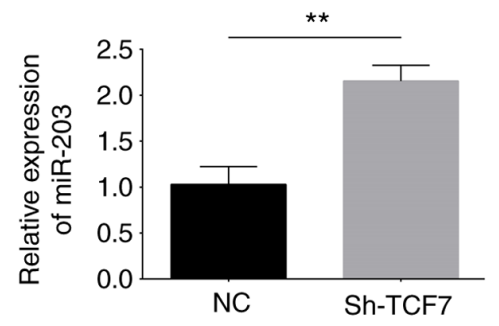

G

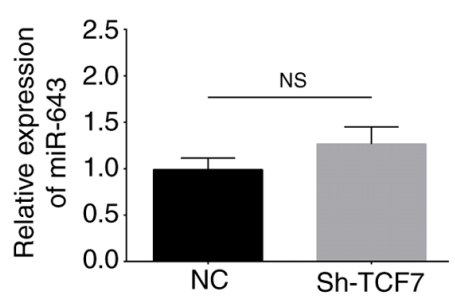

Figure 4. Cellular proliferation and apoptosis following lnc-TCF7-knockdown and potential target miRNAs of lnc-TCF7 in RPMI8226 cells. (A) 1nc-TCF7 expression was reduced following lnc-TCF7-knockdown. Cellular proliferation was suppressed at (B) 48 and $72 \mathrm{~h}$, while (C and D) apoptosis was enhanced after lnc-TCF7-knockdown. (E) miR-203 was upregulated, while (F) miR-26b and (G) miR-643 levels remained unchanged following lnc-TCF7-knockdown. P $<0.05$, ${ }^{* *} \mathrm{P}<0.01,{ }^{* * *} \mathrm{P}<0.001$. lnc-TCF7, long non-coding RNA transcription factor 7; MM, multiple myeloma; NC, negative control; miR/miRNA, microRNA.

associated with carcinogenesis (such as the Wnt pathway), thus increasing the risk of MM, as well as patient $\beta 2-\mathrm{MG}$ levels and ISS stage (7). Finally, lnc-TCF7 may serve as a gene pool for TCF7, while the loss of TCF7 diminishes hematopoietic stem/progenitor cell functions. Hence, lnc-TCF7 may promote TCF7 expression and stimulate the malignant proliferation of MM cells, thus increasing risk of MM, $\beta 2-\mathrm{MG}$ levels and ISS stage (11). The predictive value of lnc-TCF7 for the prognosis of patients with MM was subsequently evaluated, and lnc-TCF7 expression was revealed to be negatively related to EFS and OS. These results align with those of a previous study, which indicated that lnc-TCF7 is associated with poorer OS in patients with CRC (10). The negative relation between lnc-TCF7, EFS and OS in MM may be the result of lnc-TCF7 promoting MM tumorigenesis, which exacerbates clinical features and therefore promotes poorer EFS and OS. Alternatively, lnc-TCF7 may facilitate disease relapse via the activation of mesenchymal stem cells, thus promoting poorer EFS and OS in patients with MM. In addition, univariate analysis indicated that lnc-TCF7 expression level was related to reduced EFS and OS times, while multivariate analysis revealed that lnc-TCF7 was not an independent risk factor for shorter EFS or OS. These findings may be explained by lnc-TCF7 indirectly influencing survival by affecting other malignant features (such as ISS stage). Also, it was found that older age, Durie-Salmon stage and ISS stage were independent risk factors for shorter EFS, while age and Durie-Salmon stage were non-significant in the univariate analysis. Further studies should be conducted to verify these findings.
A number of in vitro studies have sought to clarify the effects and molecular mechanisms of lnc-TCF7 in cancer pathology (8-10). For instance, Wang et al (7) discovered that lnc-TCF7 is highly expressed in liver cancer stem cells (CSCs; Hep3B and Huh7 cell lines), and that its overexpression enhances the tumorigenic capacity of liver CSCs by targeting the Wnt signaling pathway (7). Similarly, Li et al (10) revealed that lnc-TCF7-knockdown decreases the migration and invasiveness of DLD1 and LoVo CRC cell lines by regulating the Wnt/ $\beta$-catenin signaling pathway, while its overexpression has the opposite effect. In addition, lnc-TCF7 promotes the invasiveness and self-renewal of NSCLC cells (A549 and 95D cell lines) by upregulating Slug and epithelial cell adhesion molecule expression (9). However, few studies had previously investigated the effects of lnc-TCF7 on the activities of MM cells. The results of the present study revealed that lnc-TCF7-knockdown inhibited MM cell proliferation while promoting apoptosis, potentially by acting as a ceRNA and sponging tumor suppressor miRNAs, such as miR-203. Alternatively, lncTCF7 may directly recruit the SWItch/sucrose non-fermentable complex to the TCF7 promoter, activating tumorigenesis-related pathways (such as the Wnt signaling pathway), and then stimulating cellular proliferation while repressing apoptosis (7). Finally, lnc-TCF7 may serve as the gene pool of TCF7, inducing the upregulation of TCF7 (which may stimulate the activities of malignant hematological cells), thereby promoting proliferation and suppressing apoptosis (11). However, further studies are required to clarify the effects of lnc-TCF7 on the migration and invasion abilities of MM cells. 

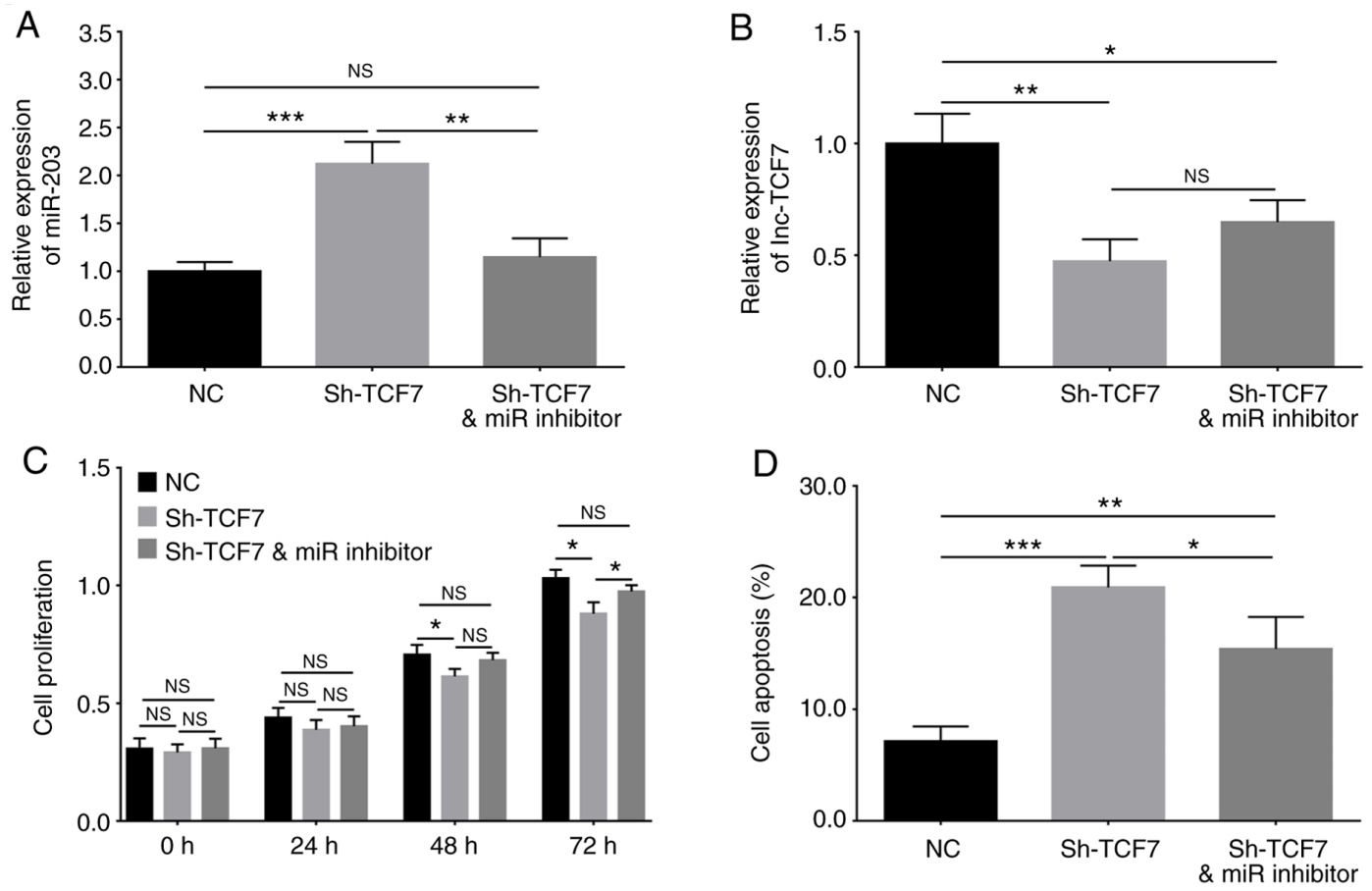

$E$

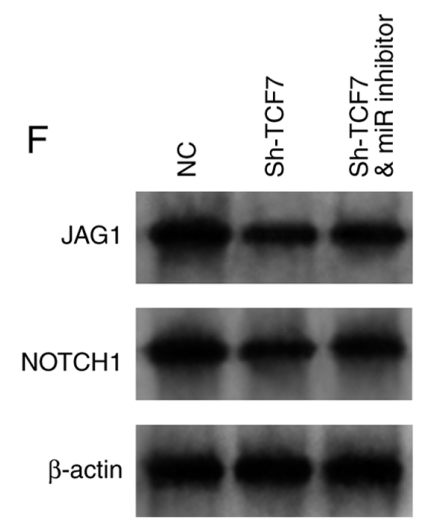

AV

G

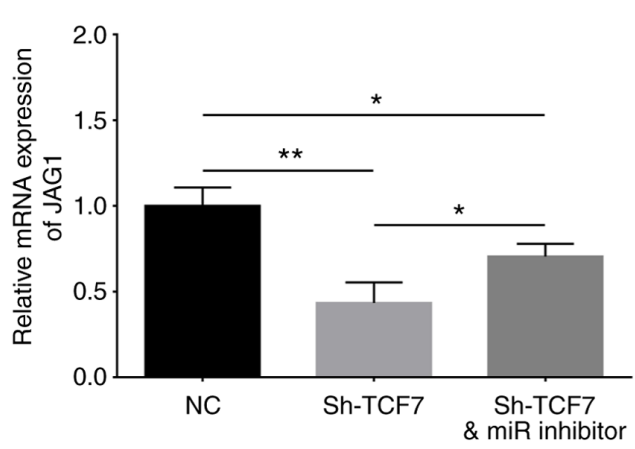

$\mathrm{H}$

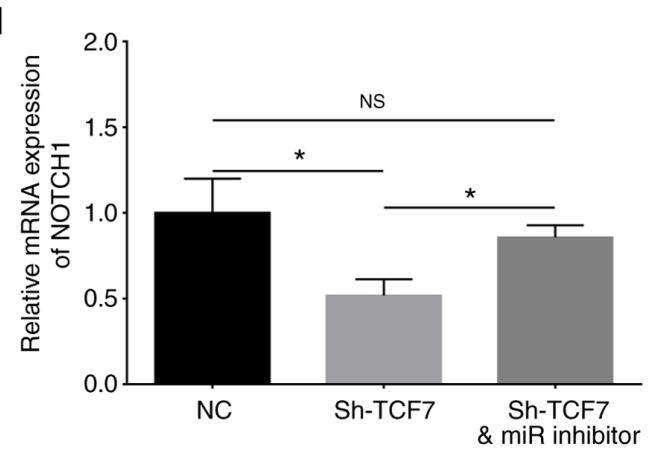

Figure 5. Interplay between lnc-TCF7 and miR-203 regulates the Jagged1-Notch1 signaling pathway in RPMI8226 cells. (A) miR-203 expression was repressed, while (B) lnc-TCF7 expression was unchanged following miR-203-inhibition in lnc-TCF7-knockdown cells. (C) Cellular proliferation was enhanced, while (D and E) apoptosis was suppressed following miR-203 inhibition in lnc-TCF7-knockdown cells. ( $F$ and $G$ ) Jagged1 and $(F$ and $H)$ Notch1 expression were enhanced by miR-203 inhibition in lnc-TCF7-knockdown cells. $\mathrm{P}<0.05,{ }^{* *} \mathrm{P}<0.01,{ }^{* * *} \mathrm{P}<0.001$. lnc-TCF7, long non-coding RNA transcription factor 7; MM, multiple myeloma; NC, negative control; sh, short hairpin; miR, microRNA.

The miRNAs are a class of non-coding RNAs of $\sim 20$ nucleotides in length that are abundantly expressed in mammalian cell types (19). In the past few decades, a large number of miRNAs have been reported to act as modulators of cancer pathogenesis (in malignancies such as lung and ovarian cancer) $(20,21)$. In MM, several miRNAs have been reported to exert anti-cancerous functions $(22,23)$. Wu et al (22) observed that miR-203 suppressed MM cell proliferation and regulated $\mathrm{G}_{1} / \mathrm{S}$ transition by targeting Bmi-1. Jia et al (23) demonstrated that miR-26b inhibited cellular proliferation and induced apoptosis by targeting Jagged1 in MM cells. These studies indicate that miRNAs serve crucial roles in inhibiting the progression 
of MM. Considering that miR-203 and miR-26b play supressive roles in MM development, and in addition to miR-643, are predicted targets of lnc-TCF7 $(22,23)$, the present study aimed to determine whether lnc-TCF7 reversely mediated these three miRNAs by evaluating their expression following lnc-TCF-knockdown. The data showed that lnc-TCF7-knockdown stimulated miR-203 expression but did not affect that of miR-26b or miR-643, suggesting that lnc-TCF7 reversely modulated miR-203 expression in MM cells. To further investigate the effects of lnc-TCF7-knockdown on MM cell proliferation and apoptosis via miR-203, the miRanda database was used to predict critical genes regulated by miR-203, which revealed Jagged1 as a potential target gene. A previous study has also reported that the Jagged1-Notch1 signaling pathway is directly regulated by miR-203 (18), thus Jagged1 and Notch1 were selected as candidate genes in further miR-203 compensation experiments in the present study. The expeiments revealed that the effects of lnc-TCF7-knockdown on MM cell proliferation and apoptosis may result from its regulation of the miR-203-mediated Jagged1-Notch1 signaling pathway. However, further rescue experiments could be conducted to investigate the regulatory role of lnc-TCF7 and miR-203 on the Jagged1-Notch1 signaling pathway. In brief, the present study provides an alternative perspective for understanding the molecular mechanisms of MM etiopathogenesis, and may facilitate the identification of novel therapeutic targets for MM treatment.

In conclusion, lnc-TCF7 is clinically valuable for predicting increased MM risk, and its elevated expression level is related to deteriorating clinical features and poor prognosis. Furthermore, lnc-TCF7-knockdown inhibits cellular proliferation while stimulating apoptosis by regulating the miR-203-mediated Jagged1-Notch1 signaling pathway in MM.

\section{Acknowledgements}

Not applicable.

\section{Funding}

This study was supported by The Key Medical Subject of Qiang Wei Project in Jiangsu Province (grant no. ZDXKB2016009), The Nantong Hematological Clinical Medical Research Center (grant no. HS2015004), The National Natural Science Foundation of China Grants (grant no. 81070400) and The Medical Innovation Team and the Leading Talent Project of Jiangsu (grant no. LJ201136).

\section{Availability of data and materials}

The datasets used and/or analyzed during the current study are available from the corresponding author on reasonable request.

\section{Authors' contributions}

HL, HH and YJ conceived and designed the experiments and confirm the authenticity of all raw data. HYL, YS and YX performed the experiments. LW, CZ and $\mathrm{LH}$ made substantial contributions to the acquisition, analysis and interpretation of data. YJ was involved in drafting the manuscript. LH revised the manuscript critically for important intellectual content. All authors read and approved the final manuscript.

\section{Ethics approval and consent to participate}

The study was approved by The Institutional Review Board of The Affiliated Hospital of Nantong University (Nantong, China). All participants or their guardians provided written informed consent upon enrollment.

\section{Patient consent for publication}

Not applicable.

\section{Competing interests}

The authors declare that they have no competing interests.

\section{References}

1. Chan HSH, Chen CI and Reece DE: Current review on high-risk multiple myeloma. Curr Hematol Malig Rep 12: 96-108, 2017.

2. Weaver CJ and Tariman JD: Multiple myeloma genomics: A systematic review. Semin Oncol Nurs 33: 237-253, 2017.

3. Siegel RL, Miller KD and Jemal A: Cancer statistics, 2018. CA Cancer J Clin 68: 7-30, 2018.

4. Rafei H, Haroun F and Tabbara IA: Novel immunotherapeutic agents for the treatment of multiple myeloma. Am J Clin Oncol 42: 317-329, 2019.

5. Riccomi G, Fornaciari G and Giuffra V: Multiple myeloma in paleopathology: A critical review. Int J Paleopathol 24: 201-212, 2019.

6. Vo MC, Lakshmi TJ, Jung SH, Cho D, Park HS, Chu TH, Lee HJ, Kim HJ, Kim SK and Lee JJ: Cellular immunotherapy in multiple myeloma. Korean J Intern Med 34: 954-965, 2019.

7. Wang Y, He L, Du Y, Zhu P, Huang G, Luo J, Yan X, Ye B, $\mathrm{Li} \mathrm{C}$, Xia P, et al: The long noncoding RNA lncTCF7 promotes self-renewal of human liver cancer stem cells through activation of Wnt signaling. Cell Stem Cell 16: 413-425, 2015.

8. Wu B, Chen M, Gao M, Cong Y, Jiang L, Wei J and Huang J: Down-regulation of lncTCF7 inhibits cell migration and invasion in colorectal cancer via inhibiting TCF7 expression. Hum Cell 32: 31-40, 2019.

9. Wu J and Wang D: Long noncoding RNA TCF7 promotes invasiveness and self-renewal of human non-small cell lung cancer cells. Hum Cell 30: 23-29, 2017.

10. Li T, Zhu J, Wang X, Chen G, Sun L, Zuo S, Zhang J, Chen S, Ma J, Yao Z, et al: Long non-coding RNA IncTCF7 activates the Wnt/ $\beta$-catenin pathway to promote metastasis and invasion in colorectal cancer. Oncol Lett 14: 7384-7390, 2017.

11. Huls G, van Es J, Clevers H, de Haan G and van Os R: Loss of Tcf7 diminishes hematopoietic stem/progenitor cell function. Leukemia 27: 1613-1614, 2013.

12. Ludwig H, Miguel JS, Dimopoulos MA, Palumbo A, Garcia Sanz R, Powles R, Lentzsch S, Ming Chen W, Hou J, Jurczyszyn A, et al: International Myeloma Working Group recommendations for global myeloma care. Leukemia 28: 981-992, 2014.

13. Durie BG and Salmon SE: A clinical staging system for multiple myeloma. Correlation of measured myeloma cell mass with presenting clinical features, response to treatment, and survival. Cancer 36: 842-854, 1975.

14. Greipp PR, San Miguel J, Durie BG, Crowley JJ, Barlogie B, Bladé J, Boccadoro M, Child JA, Avet-Loiseau H, Kyle RA, et al: International staging system for multiple myeloma. J Clin Oncol 23: 3412-3420, 2005.

15. Anderson KC, Alsina M, Atanackovic D, Biermann JS, Chandler JC, Costello C, Djulbegovic B, Fung HC, Gasparetto C, Godby K, et al: Multiple myeloma, version 2.2016: Clinical practice guidelines in oncology. J Natl Compr Canc Netw 13: 1398-1435, 2015. 
16. Kumar S, Paiva B, Anderson KC, Durie B, Landgren O, Moreau P, Munshi N, Lonial S, Bladé J, Mateos MV, et al: International myeloma working group consensus criteria for response and minimal residual disease assessment in multiple myeloma. Lancet Oncol 17: e328-e346, 2016.

17. Livak KJ and Schmittgen TD: Analysis of relative gene expression data using real-time quantitative PCR and the 2(-Delta Delta C(T)) Method. Methods 25: 402-408, 2001.

18. Zhou Z, Shu B, Xu Y, Liu J, Wang P, Chen L, Zhao J, Liu X, Qi S, Xiong K, et al: microRNA-203 modulates wound healing and scar formation via suppressing Hes1 expression in epidermal stem cells. Cell Physiol Biochem 49: 2333-2347, 2018.

19. Duchaine TF and Fabian MR: Mechanistic Insights into MicroRNA-Mediated gene silencing. Cold Spring Harb Perspect Biol 11: a032771, 2019.

20. Mihanfar A, Fattahi A and Nejabati HR: MicroRNA-mediated drug resistance in ovarian cancer. J Cell Physiol 234: 3180-3191, 2019 .
21. Wu SG, Chang TH, Liu YN and Shih JY: MicroRNA in lung cancer metastasis. Cancers (Basel) 11: 265, 2019.

22. Wu SQ, Niu WY, Li YP, Huang HB and Zhan R: miR-203 inhibits cell growth and regulates G1/S transition by targeting Bmi-1 in myeloma cells. Mol Med Rep 14: 4795-4801, 2016.

23. Jia CM, Tian YY, Quan LN, Jiang L and Liu AC: miR-26b-5p suppresses proliferation and promotes apoptosis in multiple myeloma cells by targeting JAG1. Pathol Res Pract 214: 1388-1394, 2018

(i) $($ ) This work is licensed under a Creative Commons Attribution-NonCommercial-NoDerivatives 4.0 International (CC BY-NC-ND 4.0) License. 